\title{
Motor Activity in the Development of the Individual's Divergent Thinking and Self-esteem
}

\author{
Manue la Vale ntini ${ }^{1}$, Giovanna Troiano ${ }^{2, *}$, Simona Balzano ${ }^{3}$ \\ ${ }^{1}$ Department of Human Sciences, University of Urbino Carlo Bo, via Mainardi, 16, 61029, Urbino (PU) Italy \\ ${ }^{2}$ University of Urbino Carlo Bo, via Vitruvio, 76, 04023, Formia (LT) Italy \\ ${ }^{3}$ Department of Economics, University of Cassino, via S.Angelo, 03043, Cassino (FR) Italy
}

\begin{abstract}
Movement is a biological need which from early childhood plays a fundamental role in proper functional, physiological and mental development. It has been widely shown that independence at the level of action leads to individual's achievement and impacts upon cognitive and affective behaviour, enforcing autonomy and self-esteem. The aim of this paper is to verify the effect of motor activity on the development of children's divergent thinking and self-esteem by using an experimental study. This study was carried out within a project investigating creativity, fantasy and movement in nursery school children, and is based on a so-called ludic motor approach. The experiment was designed in the context of laboratory activities, helping to translate knowledge into skills, within the motif of the "Peter Pan adventures" film. Referring to educational research methodology, we observed 22 five-year-old children divided into a working group and a control group. Each experimental session was organized in four stages: contextualization, warm-up, middle stage and cool-down. The children's skills were measured by extensively validated tests, whose results were analy zed by statistical analysis of variance, with a view to ascertaining whether mean scores differ under different conditions. We conclude that the experiment confirms our research hypothesis that motor activity has a positive effect upon divergent thinking and self-esteem.
\end{abstract}

Keywords Children, Movement, Creativity, Self-esteem

\section{Introduction}

The importance of the motor aspect as regards the development of single capabilities already emerges during gestation. Before being a sensory organism the embryo is first of all a moving organism. Only a few weeks after birth the infant displays an interactive synchronicity, a "dance" made up of micro movements in response to human language. The movements will become increasingly complex and will appear in sequence, giving life to actions performed by memo ry in response to specific, stereotyped situations, hence termed scripts[22]. It has been proved that the level of independence impacts upon achievement and affects $\operatorname{cog}$ itive and affective behaviour, favouring greater self-esteem, increasing self-efficacy, i.e. the capacity to reach set goals, and offering useful material to build and fortify self-esteem. There fore we can conclude that our brain is an enormous data repository of motor repertories[3]. During its evolutionary process, the brain needs to have tactile and motor experiences in order to develop those sensory-motor areas that represent the starting point for the development of the superior areas, such as language and

* Corresponding author:

giovy.tro@libero.it (Giovanna Troiano)

Published online at http://journal.sapub.org/edu

Copyright (C) 2012 Scientific \& Academic Publishing. All Rights Reserved complex thinking. In its initial phase the infant's mind is above all concrete, based on direct interaction. Maria Montessori thus termed it an absorbent mind[14], which learns by means of a sort of immersion in a sensorial bath where gestures, positions, movements and emotions make learning motivating and effective. Hence, it is important to stress the great usefulness of physical activity on the different motor, cognitive, psycho-emotional and social levels, especially during early childhood[1].

In particular, between 3 and 5 years of age, motility and learning are closely related: children walk, run, move awkward ly and do not know all the parts of their bodies, but with experience they harness a greater motor capacity[16]. In the first years of life, movement is a biological necessity that assumes enormous importance for proper functional, anatomical, physiological and mental development of every individual, as well as for periodically maintaining the capacities achieved[12]. Hence, an education system that places motor activity at its core will always be less verbal and more stimulating, and will let actions speak as a factor related to a set of body signals.

Furthermore, in our current ever-changing society there is a constant need for individuals who are ready for change and above all competent. We refer to the capacity of meta-reflection on one's own cognitive processes because only in this way can we reach significant learning[19]. Experience is ideal for developing skills, so me of which are 
especially useful to manage new problems and complex undertakings that require personal initiative, imagination, self-control, reflectiveness and method[6]. Thanks to educational interventions that break with traditional teaching methods to introduce the student to new settings, through experience the child comes into contact with new knowledge which, if presented and acquired in investigative and thought-provoking contexts like those of a laboratory, he/she transforms into learning skills[9]. Thus the child matures, entering into a growth mechanism that is both mental and physical. The child will possess reflexive thought, hence of metacognitive ability and greater confidence in handling various situations, even problems. The child will also possess a body which is in harmony with itself and the surrounding environment[19]. The child will know how to move with confidence and his/her physical nature will not be an obstacle to discovering the world, to relating to it and to expressing his/her interiority, which is an inestimable asset. A tool that allows these objectives to be achieved is undoubtedly the so-called ludic-motor laboratory ${ }^{1}[5]$. This is a particular form of workshop which, in the teaching environment, allows children to undergo continuous experimentation, acting directly on their cognitive and motor faculties. The approach has been shown to improve such faculties and the children in question learn their own limits through continuous confrontation with experience[10]. The workshop transforms pupils into individuals who are alert and ready to broaden their horizons of knowledge by way of various experiences[23].

\section{Objective of the Study}

The aim of this work was to analyse specifically how motor activity affects the development of the individual's divergent thinking and self-esteem with an experimental study. This study was carried out within the project "Creativity, fantasy and movement in nursery school children" and uses a ludic-motor approach, given that a laboratory often helps translate knowledge into skills.

The education system plays a vital role in children's development, in that it can and should promote significant interventions to optimise motility and divergent thinking, seen as factors that sustain the development of a positive self-image. Such conditions can offer the child the possibility of living serenely in the present, but mainly of looking towards a bright future.

\section{Method}

\subsection{Participants}

In all, 22 five-year-old nursery schoolchildren took part in the project. This age group was deemed preferable mainly due to the need to select a specific target audience, and also because the three to four year olds were inserted in specific courses. The latter age group was involved in entry and adaptation tasks and the former age group in educational activities that aimed towards greater development of autonomy. During the first two years of nursery school, children improve their motor capacity, but they are still very tied to exploring the environment, which does not permit complete motor autonomy. Given these reasons, it would certainly have been interesting, though more difficult, to administer tests or have exercises carried out which led to an evaluation of the gross motor skills and cognitive abilities, such as those taken into consideration in the research. On the other hand, at five years of age a child reaches complete autonomy of movement and expression, which develops into an always greater expressive capacity, to the point of having rich, correct and well comprehensible language skills. These evolutionary characteristics allowed less problematic selection of a sample for the research and more straightforward test administration.

After the informed consent of their parents, the children were divided into two groups: the work group, which participated in all the project activities, and the control group, which was co-protagonist only during admin istration of the tests [4]. The two groups had the same characteristics of age, sex and number of participants: both groups were made up of 11 children, but the work group was made up of 6 males and 5 females, whereas, the control group was made up of 5 males and 6 females. The subdivision of the students into two groups was made by lot in order to ensure fullobjectivity of the procedure and research.

All the tests were administered to both groups in three sessions: at the beginning (October 2010), in the middle (December 2010) and at the end (April 2011) of the whole procedure. In particular, the first tests were used to assess the children's characteristics, ongoing tests to verify progress, and the final tests to ascertain experiment efficacy and compare the improvements in the work and control groups.

\subsection{Me as ures}

In the research hypothesis, two keywords, namely motility and creativity, were presented, and the objective was to establish a connection between these two factors, in that the former should influence the latter. It was thus necessary to select two types of tests, one in reference to cognitive-motor objectives and the other in reference to affective-relational objectives.

We chose, above all, tests dealing with the integration of the disabled, learning difficulties, support, psychology and social work through the production of books, magazines, educational software and on-line services. There are many catalogue tests, but in making an accurate selection and constantly bearing in mind the objectives and the target audience, we identified the following tests which have been internationally validated:

1. Test of Gross Motor Development (TGMD)[21]. This test is administered individually and evaluates the dynamic aspects of 12 gross motor skills and the movement patterns of 3- to 10-year-old children. The abilities are grouped into 
two subtests, each evaluating a different aspect of gross motor development: locomotion and object control. In particular, the first measures seven abilities: running, galloping, hopping, leaping forward, long jump from a still position, hopping forward or sideways and rolling sideways. These are all abilities that shift the centre of gravity from one point to another in space. The second subtest measures five abilities: hitting a ball with a racket, dribbling a ball from a still position, catching a thro wn ball with your hands, kicking the ball while running and throwing the ball with one hand. These are all abilities connected with throwing and receiving objects, which is a particularly evolved form of object control.

2. Creativity Assessment Packet (CAP)[24]. This test is administered individually and permits evaluation of eight factors of divergent thinking and creative personality. Thanks to the tools and procedures of this test, it is possible to diagnose the level of creativity of the student's performance and indicate the factors of divergent thinking and creative personality, which are more important for the development of creativity. The creativity test was initially elaborated to select particularly gifted children in those schools that provided specific programs for the development of creative capacities. Now, instead, it is available to measure the creativity potential of all children. The CAP consis ts of three different tools: the Divergent Thinking Test and the Divergent Feeling Test, both administered to subjects fromabout five years of age to 18 years of age, the Williams Scale Test, for children to be evaluated by parents and teachers. Specifically:

2.1. Divergent thinking test (Protocols $A$ and $B$ ): this test measures a combination of verbal capacities that depend on the left hemisphere of the brain and the non-verbal visual-perceptive capacities that depend on the right hemisphere of the brain. The test consists in assigning scores for the cognitive factors of divergent thinking fluency, flexibility, originality, elaboration, title, derived from Guilford's human intelligence theory[8]. The underlying processes that are evaluated are divergent transformations of figures (v isual creativity) and giving names to the produced figures, which requires verbal capacity (divergent semantic transformation). Protocols $\mathrm{A}$ and $\mathrm{B}$ differ in the kind of figures given to the subjects, asking them to elaborate them and to create on that starting basis their new ones. The test thus solicits contemporaneous processing of the right and le $\mathrm{ft}$ brain hemisphere which are, therefore, at the same time cognitive and emotional.

2.2. Divergent Feeling Test (DFT). This test is a list of 50 multiple choice items to ask children how curious, imaginative, attracted to complexity and inclined to accept risks they think they are. The test gives both a total score and four sub scores related to the affective factors curiosity, imagination, challenge and risk-taking, all aspects of the emotional nature, which include both verbal analysis and emotional processes (left and right brain hemisphere).

2.3. Williams Scale. This consists of an evaluation form that refers to the observation of the eight factors related to the creativity measured by the tests. It presents six characteristics for each of the eight factors for which parents and teachers are asked to express an evaluation, in relation to the child. The scale gives a rough score for 50 multiple choice items, while the open sentences can be analysed and evaluated on the bas is of the frequency with which they are marked in a group of parents or teachers, for a particular class or given group of students. This scale indicates the level of each creativity factor that the observed child possesses, and can also be useful for good attitudinal evaluation of a creative child by the parent or teacher.

In order to achieve affective-relational objectives, it was deemed appropriate to also present the pupils with educational proposals aimed towards forming sound self-esteem, a fundamental factor for everyone and especially for children: feeling good about thems elves allows even young children to learn more effectively since it helps them to better face the tensions of life and encourages them to create a better future. In order to achieve such objectives, we referred to the Plummer approach[15], which offers more than 100 simple, practical and fun activities, specifically aimed towards helping children to build and maintain a good level of self-esteem. Based on Plummer's vast clinical experience, these activities encourage children to use their imaginative and naturally creative abilities in considering their relationship with themselves, with their families, with their friends, with their world, and to express feelings in words or through images. This course was carried out with motor-play atelier activities since a creative child is also more self-confident, and hence less controlled in expressing his fantasies, curiosities and desires. The forms that refer to this tool are analytically presented.

At the end of each encounter, a satis faction survey of the daily proposed activities was taken. In a moment of final reflection, precisely when we were all sitting in a circle, we asked the children to remain still if they had not enjoyed themselves, to clap their hands if they had enjoyed themselves, and to clap their hands and stomp their feet if they had enjoyed themselves very much. During the last encounter, we also asked the children to make a drawing that showed the various phases that were followed, the persons encountered, and the emotions felt, in order to have greater confirmation of the shared experience.

In conclusion, once the cycle of sessions with the children was finished, the parents were given a satisfaction questionnaire. This asked them, in a synthetic way, to give their impression of the project presentation and its execution, as well as any changes in the children concerning the motor and creative area of interest.

\subsection{Proce dures}

The educational interventions were structured into two learning units: "Run, jump, play and fly with your fantasy..." and "...Together the treasure can be won!". These are linked to the contents, but above all to the background supplement that functioned as a contextualizing framework and which made reference to the animated film Peter Pan. Each unit 
made reference to the following key points: identification of the starting situation; identification of the objectives; definition of the contents; definition of the methodology; definition of methods and tools; systematic observation of the learning processes; verification; evaluation. In particular, play methodology was used as the game proved an excellent tool to respond to the natural curiosities and interests of the children.

Each session was subdivided into different stages: the contextualization stage, in which the children watched part of the animated film; the warm-up stage, in which, after a demonstration, the children carried out movements in succession and muscle-stretching exercises; the middle stage, in which motor courses and creative activities were used, such as the construction of binoculars for exploration on the fantasy island; the cool-down stage, where motor games were mainly carried out, a mong which the most popular were those that used music, an element that proved an excellent communication tool. Based on the type of activity, it was possible to use different environments: the hall with the equipment for motor activity, the video room to see the animated film and the library for creative and manual activities.

In the beginning, it was thought opportune to gradually insert ourselves into the school context, in order to analyse it and get to know the child ren. In September, we worked side by side with the teachers in their daily activities, collaborating and getting to know the surroundings that would host the research work. Furthermore, before beginning the experimental course with the work group, it was important to offer a moment to have both groups meet since they were the participants in the project. Despite only being involved in the administration of the tests, the control group achieved results that led to a comparison and reflection of a scientific, educational and formative nature, which generated favourable results for our research. For these reasons, mo ments of shared play, for the work group and the control group, were programmed so as to help all the schoolmates get acquainted; the socialization process among the children continued during the subsequent stages of the project. The two subject groups were brought together during the last encounter at the final party prepared by the work group children, and the entire school complex participated. The event was organized so as to involve the entire school context, where the project was carried out over the course of months. Indeed, this event cannot be isolated from the educational framework, in the perspective of formative continuity aiming towards the development of each student, in respect to his/her peculiarities and potential, but also in respect of the environment, which comprises structures and persons where the student is integrated on a daily basis.

\subsection{Statistical Analysis}

Statistical analysis aimed at comparing the test results of the work and control groups, which means testing the hypothesis that mobility and motility improve children's divergent thinking and self-esteem[13].

All test results are expressed on a numeric scale, then the analysis was based on the Analysis of Variance (ANOVA) method, according to the one-way ANOVA scheme[17]. According to this scheme, we measure the effect of one factor $A$ (mobility and motility) on a response variable $Y$ (creativity and self-esteem) observed on $n$ statistical units. In particular, we seek to assess to what extent belonging to the work or control group (factor $A$ ), i.e. participating in all the project activities or otherwise, affects children's performances in the TGMD and CAP tests (response variable $Y$ ).

The general theory underlying the ANOVA model can be summarised as follows. Supposing that $k$ treatments of factor $A, A_{1}, A_{2}, \ldots, A_{i}, \ldots, A_{k}$ are given to the $n_{1}, n_{2}, \ldots, n_{i}, \ldots, n_{k}$ units $\left(n_{1}+n_{2}+\ldots+n_{i},+\ldots+n_{k}=n\right)$ and that $y_{i j}$ is the response of $j$-th unit $\left(j=1, \ldots, n_{i}\right)$ to the $i$-th treatment, the experiment provides the synopsis of results shown in table 1.

The statistical issue is to test the equality of means among treatments (null hypothesis), meaning that factor $A$ has no effect upon the response variable, against the alternative hypothesis that means are different, meaning that factor $A$ has some effect on $Y$. Accepting the null hypothesis (i.e. if $p$-value $>0.05)$ we assess that there is no effect, while rejecting it (i.e. if $p$-value $<0.05$ ) means that the effect is significant. In conclusion, we can interpret results by considering $p(0 \leq p \leq 1)$ as inversely related to the effect.

With respect to each factor a parallel analysis aimed at testing the repetition effect, i.e. whether there is a difference in children's performance among the three repetitions of the tests. To this aim, consistent with how data were collected, we followed the repeated measures ANOVA scheme[7], where it is assumed that measures on the same subject at different times are not independent.

Table 1. ANOVA data structure: summary of results of a k treatments experiment, mean response to each treatment in the last column

\begin{tabular}{ccc|}
\hline Tre atment & Response & Mean \\
$\mathrm{A}_{1}$ & $\mathrm{y}_{11}, \mathrm{y}_{12}, \ldots, \mathrm{y}_{\mathrm{nn}_{1}}$ & $\bar{y}_{1}$ \\
$\mathrm{~A}_{2}$ & $\mathrm{y}_{21}, \mathrm{y}_{22}, \ldots, \mathrm{y}_{2 \mathrm{n}_{2}}$ & $\bar{y}_{2}$ \\
$\vdots$ & $\vdots$ & $\vdots$ \\
$\mathrm{A}_{\mathrm{k}}$ & $\mathrm{y}_{\mathrm{k} 1}, \mathrm{y}_{\mathrm{k} 2}, \ldots, \mathrm{y}_{\mathrm{kn}_{\mathrm{k}}}$ & $\bar{y}_{k}$ \\
\hline
\end{tabular}

\section{Results}

The following sections show results from both case-control analysis and repetition effect analysis (among the three repetitions) for each test: TGMD, Divergent Thinking Test (Protocol A and B), Divergent Feeling Test and William scale.

For all tests we report only significant effects.

\subsection{Test of Gross Motor Development (TGMD)}


The TGMD score (Figure 1) exhibits significant differences in both case-control scores $(p=0.013)$ and repetition $(\mathrm{p}<0.0001)$.

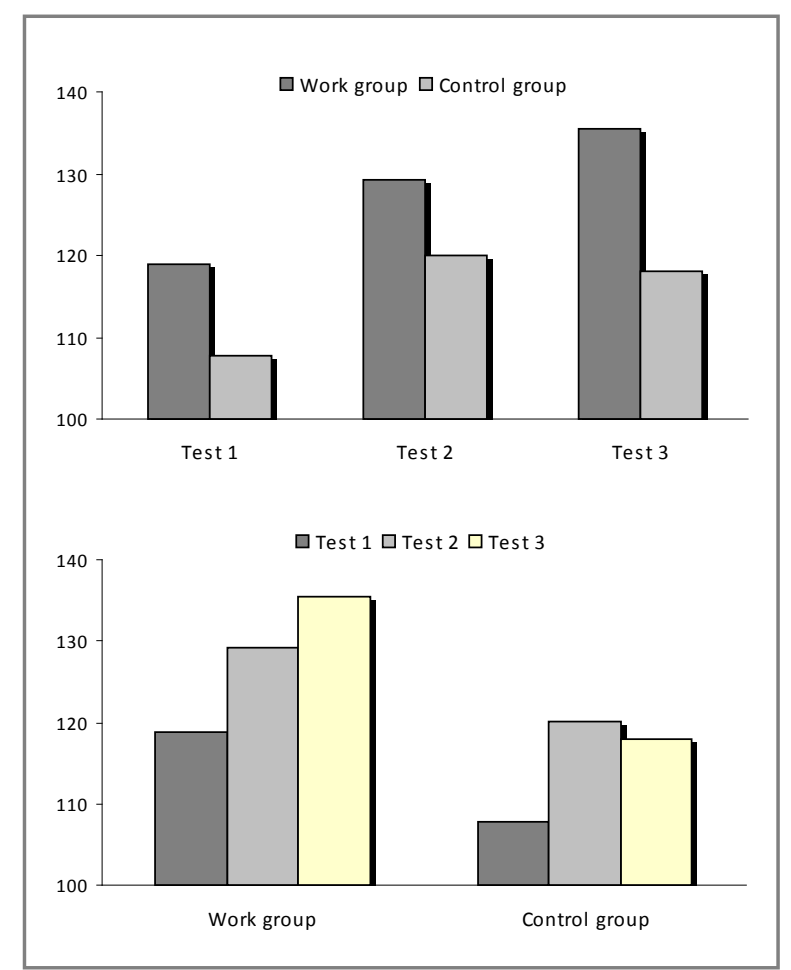

Figure 1. Mean TGMD score: case-control effect and repet ition effect

In particular, the work group score is always higher than control group, meaning a significant effect of the activity of the work group. On the other hand, in both work and control group the performance generally increases from one test to the next, showing a significant effect of the repetition of the exercise. Nevertheless, this effect is more evident in work than in control group, as in the former the performance constantly improves while in the latter only a partial improvement comes out.

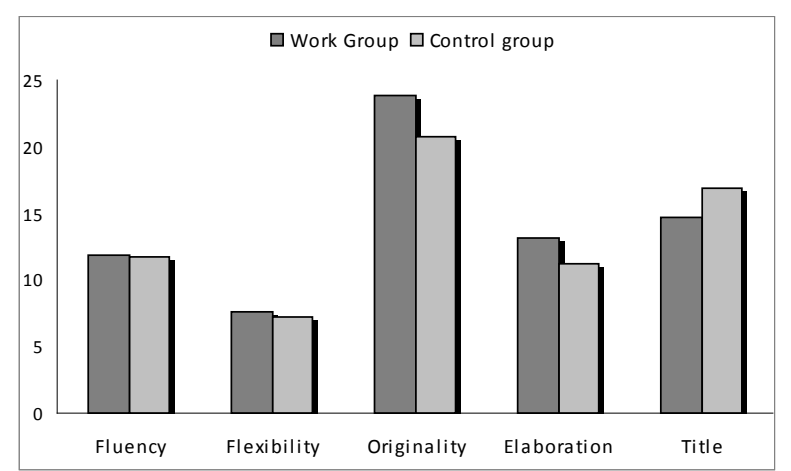

Figure 2. Protocol A mean scores for case and control groups (factors: Fluency, Flexibility, Originality, Elaboration, Title)

\subsection{Divergent Thinking Test - Protocols A and B}

Work and control groups were compared by Protocol A and $\mathrm{B}$ tests with respect to fluency, flexibility, orig inality, elaboration, title. For the Protocol A test (Figure 2) significant differences were found between scores for originality $(p=.021)$ and flexibility $(p=.033)$. No repetition effect was detected for Protocol A.

For the Protocol B test (Figure 3) significant differences were found between scores for fluency $(p=0.026)$ and originality $(p=0.000)$. Repetition effect was detected only for factor elaboration $(\mathrm{p}=0.002)$.

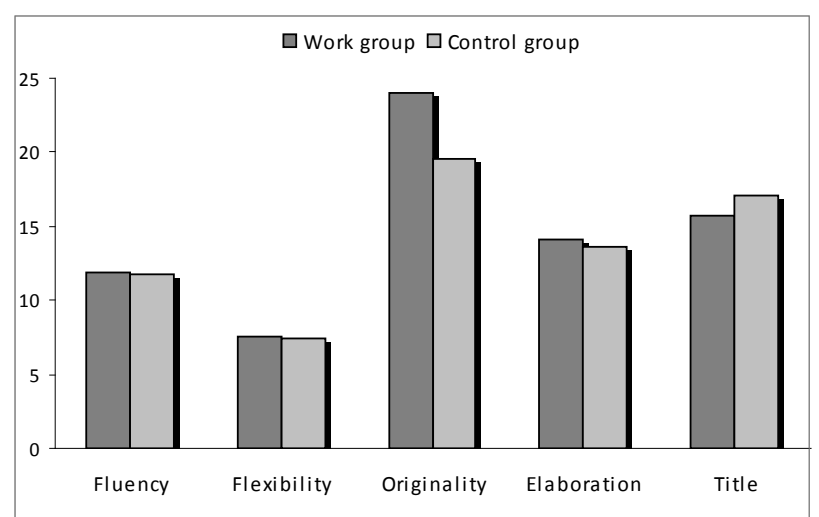

Figure 3. Protocol B mean scores for case and control groups (factors Fluency, Flexibility, Originality, Elaboration, Title)

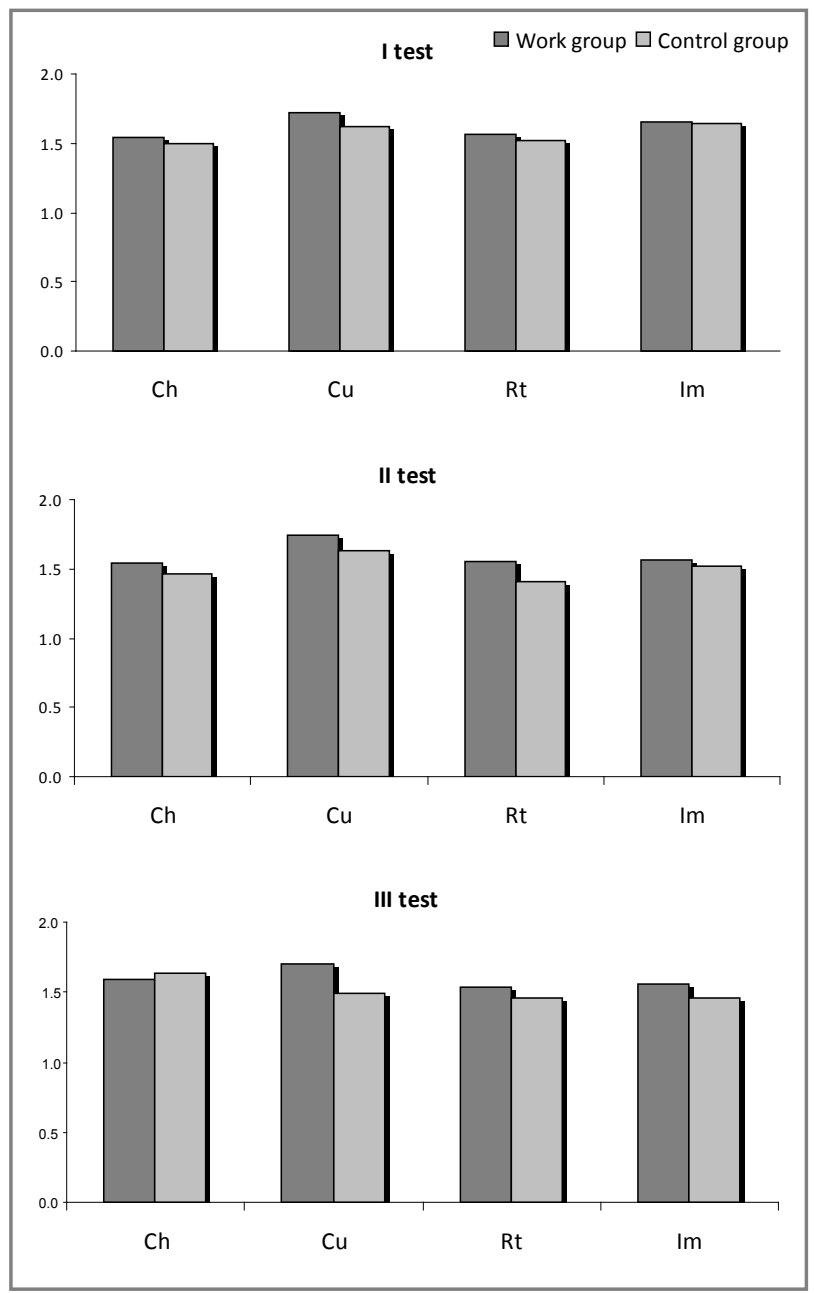

Figure 4. Mean DFT scores in the three tests for case and control groups for factors: attraction to challenge $(\mathrm{Ch})$, curiosity $(\mathrm{Cu})$, risk-taking $(\mathrm{Rt})$, imagination $(\mathrm{Im})$

\subsection{Divergent Feeling Test (DFT)}


Together with the case-control study, we analysed DFT data according to a different perspective as well: the test of the effect of each specific observed factor of creative personality: attraction to challenge $(\mathrm{Ch})$, curiosity $(\mathrm{Cu})$, risk-taking (Rt), imagination ( $\mathrm{Im})$. In the latter analysis, significant differences were found for the factor curiosity ( $p$ $=0.045)$. Moreover, multiple test analysis for paired comparison[18] shows significant differences between factors $\mathrm{Cu}$ vs $\operatorname{Dr}(p=0.003)$ and $\mathrm{Cu}$ vs $\mathrm{Co}(p=0.030)$. Figure 4 shows the case-control comparison for the DFT score (all factors put together) in the three repeated tests. The effect of each factor was analysed within case and control groups separately as well as in the whole group (work + control). No significant effect was found for any factor in any of the three groups.

\subsection{Williams Scale}

Among all factors, represented in Figure 5, significant differences were found between scores of imag ination ( $p=$ $0.058)$, elaboration $(p=0.012)$ and originality $(p=0.001)$.

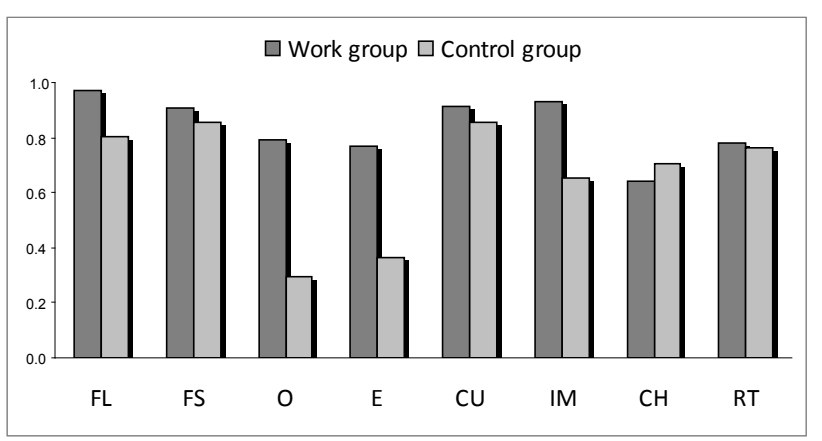

Figure 5. Williams scale mean scores for case and control groups for factors: fluency $(\mathrm{FL})$, flexibility $(\mathrm{FS})$, originality $(\mathrm{O})$, elaboration $(\mathrm{E})$, curiosity (CU), imagination (IM), attraction to challenge (Ch), risk-taking (Rt)

\section{Discussion}

Analysis of the results obtained from admin istration of the tests clearly provided positive verification with respect to the assumed expectations at the beginning of the research. The work group reached the preset objectives since it participated in the interventions aiming towards acquisition, development and growth of motor-cognitive abilities, that are influential affective-relational factors. In the previous description, the factors that showed significant score differences are especially highlighted, but in examining the graphs it clearly emerges that the results of the work group are better than those of the control group. It can be inferred from this observation that movement is a factor that significantly contributes to the development of other abilities typically linked, as in this specific research, to divergent thinking. The factors considered are interconnected and mutually influence one another. Hence it is possible to offer a solution to the research hypothesis: motor activity positively influences the development of divergent thinking and self-esteem.
With this statement, the importance of movement, as a factor that considerably influences the cognitive, emotional and relational factors, appears even more significant. This tool is indispensable and must not be forgotten when dealing with educational approaches. Hence, in the educational field, selecting dynamic intervention methods, linked to the motor area through stimulating approaches, such as the project "Creativity, fantasy and movement with nursery school children", will allow children to achieve formative objectives and significant learning that will sustain their growth. Indeed, children that believe in themselves, in their own resources and develop a certain level of autonomy will be more prepared to face and overcome the inevitable difficulties of life, and will not always depend on others for their self-definition. In respect of the psychophysical development of the child, movement assumes a significant role[20]. When inserted into an organized, well planned and above all stimulating context, that follows a specific program, the student becomes the protagonist of truly significant formative it ineraries. These lead to g lobal growth where the person learns by doing. Educational activity is much more than a refined transmission of information. It is something that is physical and interpersonal. Hence, the structural elements of this type of educational planning are the exploration of physical nature, through the discovery of the significance of gestures, in which learning processes can develop through exploratory moments in performing research and experimentation[9].

\section{Conclusions}

Through movement, individuals learn to explore the environment in which they live, and learn to commun icate. Control of bodily self-perception reinforces the image that we all have of ourselves[11]. After all, the skin, not only the derma, is a means of osmotic communication between something external towards something internal and vice versa. At the same time, it is a sensitive boundary, a demarcation that distinguishes us and defines the natural site of an affective relationship, of pleasure and pain and of meeting and loss. We can forget the body, but the body cannot forget us[2].

Let us not enclose the fantasy and abilities of children in cages that offer only ready-made alternatives. Let them $d o$, so that the child becomes an autonomous, secure, and respectful person, with a good self-image, a good image of others and of the world. In so doing, we will favour the birth of open, positive and propositional citizens that live their experiences rather than merely survive. The subject must become an agent. Hence, in the educational context, it is fundamental to sustain children to have them become individuals that trust in themselves and others, not in a naive way but on the basis of values that serve as a guide towards an illuminated path. This path must, therefore, be characterized by valid points of reference and not abandoned in the dark, thus leaving everyone uncertain as to which path 
to follow. Could this be utopia? No, but a difficult objective to reach if we embark on professional and life paths that lack drive, enthusiasm, motivation and dreams. Of course, these last words must be linked to reality, in order not to run the risk of losing touch with the world and alienating oneself in dangerous fantasies, but they must not be written off.

\section{Notes}

1. We use this term to describe an approach where learning is achieved by combining movement (motor) with playing (ludic).

\section{REFERENCES}

[1] Bril B., Culture et premières acquisitions motrices: enfants d'Europe d'Asie et d'Afrique, Journal de Pédiatrie et Puériculture, 10 (5), 1997.

[2] Davis C.L., Tomp orowski P.D., Boyle C.A., Waller J.L, Miller P.H., Naglieri J.A, et al., Effects of aerobic exercise on overweight children's cognitive functioning: A randomized controlled trial, in: Research Quarterly for Exercise and Sport, Auburn, 2007.

[3] Dixon M.A., Warner S.M., Bruening J.E., More than just letting them play: parental influence on women's lifetime. Sport involvement, Sociology of Sport Journal, 25, 2008.

[4] Dunham P.J., Research methods in psychology, Harper-Row, New York, 1988.

[5] Frabboni F., Il laboratorio, Laterza, Roma-Bari, 2004.

[6] Gardner H., Frames of mind. The theory of multiple intelligences, Basic Books, New York, 1993.

[7] Girden E.R., ANOVA Repeated Measures, Sage University Paper, Thousand Oaks, 1992.

[8] Guilford J.P., Creativity: retrospect and prospect. Journal of Creative Behaviour, 4 (2), 1970.

[9] Harrison N.S., Understanding Behavioral Research, Wadsworth, Belmont, 1979.
[10] Harter S., The determinants and mediation role of global self-worth in children, in Contemporary topics in developmental psychology, Wiley, New York, 1987.

[11] Heider F., The psychology of interpersonal relations, Wiley, New York, 1958.

[12] Martin D., Sportlichen Leistungsfähigkeit bei Kindern, Sportwissenschaft, 12 (3), 1988.

[13] McIver J.P., Carmines E.G., Unidimensional Scaling, Sage, London, 1981.

[14] MontessoriM., La mente del bambino, Garzanti, Milan, 1966.

[15] Plummer D., La mia autostima. Attività di sviluppo personale per una buona immagine di sé, Erickson, Trento, 2008.

[16] Rieder H., Bedeutung und Schulung von psychomotorischen Fähigkeiten für den jugendlichen Wettkampfsportler, Leistungssport, 24 (3), 1979.

[17] Sahai H., Ageel M.I., The Analysis of Variance, Birkhaüser, Boston, 2000.

[18] Scheffe, H.., A Method of Judging All Contrasts in the Analy sis of Variance, Biometrika, 40, 87-104, 1953

[19] Schunk D.H., Hanson A.R., Cox P.D., Peer model attributes and children's achievement behaviors, Journal of Educational Psychology, 79 (1), 1987.

[20] Slutzky C.B., Simpkins S.D., The link between children's sport participation and self-esteem: exploring the mediating role of sport self-concept, Psychology of Sport and Exercise, $10(3), 2009$.

[21] Ulrich D.A., TGMD Test of Gross Motor Development, PRO-ED, Austin, Texas (USA), 1985.

[22] Wallon H., Comment se développe chez l'enfant la notion du corps propre, Enfance, 1-2, 1963.

[23] Weiner B., A theory of motivation for some classroom experiences, Journal of Educational Psychology, 71 (1), 1979.

[24] Williams F., Creativity Assessment Packet, PRO-ED, Austin, Texas, USA, 1993. 